\title{
Prevalence of and risk factors for severe cognitive and sleep symptoms in ME/CFS and MS
}

Vageesh Jain ${ }^{1 *}$, Amit Arunkumar ${ }^{2}$, Caroline Kingdon $^{3}$, Eliana Lacerda $^{3}$ and Luis Nacul ${ }^{3}$

\begin{abstract}
Background: There are considerable phenotypic and neuroimmune overlaps between myalgic encephalomyelitis/ chronic fatigue syndrome (ME/CFS) and multiple sclerosis (MS). While the precise aetiologies of both MS and ME/ CFS are unclear, evidence suggests that deterioration in cognitive function is widely prevalent in patients with either condition. Little is known about differing risk factors or exposures, which may lead to severe cognitive or sleep symptoms. This study aims to gauge the extent of cognitive and sleep symptoms in ME/CFS and MS patients participating in the UK ME/CFS Biobank and identify the characteristics of those experiencing severe symptoms.
\end{abstract}

Methods: This was a cross-sectional study of 395 UK ME/CFS Biobank participants, recruited from primary care and the community, using similar standardised protocols, and matched by age, sex and geographical area. Data were collected from participants using a standardized written questionnaire at clinical visits. Cognitive symptoms included problems with short-term memory, attention, and executive function. Sleep symptoms included unrefreshing sleep and poor quality or inadequate duration of sleep. All participants reported symptoms based on an ordinal severity scale. Multivariable logistic regression was carried out in the ME/CFS group to investigate socio-demographic factors associated with severe symptoms.

Results: All cognitive and sleep symptoms were more prevalent in the ME/CFS group, with 'trouble concentrating' (98.3\%) the most commonly reported symptom. Severe symptoms were also more commonly reported in the ME/CFS group, with 55\% reporting 'severe, unrefreshing sleep'. Similarly, in the MS group, the most commonly reported severe symptoms were sleep-related. Logistic regression analysis revealed that ME/CFS patients aged over 50 years were more than three times as likely to experience severe symptoms than those younger than 30 (OR 3.23, $p=0.031$ ). Current smoking was associated with severe symptoms, increasing the risk by approximately three times (OR 2.93, $p=0.003)$ and those with household incomes of more than $£ 15,000$ per year were less likely to experience severe symptoms compared to those earning less than this (OR 0.31, $p=0.017$ ).

Conclusions: Cognitive and sleep symptoms are more common in ME/CFS patients than in MS patients and healthy controls, providing further support for existing evidence of central nervous system abnormalities in ME/CFS. Our findings suggest that people with ME/CFS who are smokers, or have a low income, are more likely to report severe cognitive and sleep symptoms. Future research should aim to develop strategies to prevent the progression of severe cognitive and sleep symptoms through early interventions that prioritise patients identified as being at highest risk.

Keywords: ME/CFS, MS, Risk factors, Cognitive symptoms, Sleep, Severity

\footnotetext{
* Correspondence: vageesh.jain@kcl.ac.uk

'Guy's Campus, King's College London School of Medicine, London SE1 1UL, UK

Full list of author information is available at the end of the article
} 


\section{Background}

Myalgic encephalomyelitis/chronic fatigue syndrome (ME/CFS) and multiple sclerosis (MS) are chronic neurological disorders characterised by a number of symptoms including debilitating fatigue and cognitive decline $[1,2]$. Some authors have proposed that ME/CFS and MS may share common aetiological and pathophysiological mechanisms [3-7]. While the precise aetiologies of both MS and $\mathrm{ME} / \mathrm{CFS}$ are unclear, evidence suggests that changes in cognitive function are a core component of the conditions $[8,9]$. Cognitive dysfunction has an impact on everyday life and reduces perceived quality of life [10] and includes problems with short-term memory, concentration, attention, and processing, which often coincide with sleep symptoms. Sleep disturbance is commonly reported, and may have an impact on non-cognitive symptoms such as fatigue, in both ME/CFS and MS [11, 12].

There are considerable phenotypic and neuroimmune overlaps between ME/CFS and MS [5, 13]. The pathophysiological process underlying changes in cognitive function in MS relates to the development of plaques affecting the myelin sheath and therefore axonal transmission [9]. The underlying process leading to cognitive changes in ME/CFS is not known, but inflammatory oxidative and nitrosative stress pathways may play a role [14].

A recent appraisal highlighted an increasing body of literature evidencing neurocognitive impairment in people with ME/CFS [1]. The authors acknowledged that despite the lack of evidence on objective changes in sleep architecture, unrefreshing sleep is omnipresent among those patients [1]. Thus, further insight into the severity of cognitive features in ME/CFS and MS will improve future diagnosis, case stratification, and management of these conditions.

Little is known about socio-demographic, behavioural and other exposures that may lead to severe cognitive and sleep symptoms, apart from potential links with life stress, which have been reported in distinct diseases [15, 16]. Consequently, Identifying risk factors for severe cognitive and sleep problems may aid in the development of methods to limit or prevent such debilitating symptoms.

This study aims to ascertain the prevalence of severe cognitive and sleep symptoms, and factors increasing the likelihood of reporting severe symptoms, in healthy controls and people with ME/CFS and MS participating in the UK ME/CFS Biobank.

\section{Methods}

\section{Population and size}

This was an analytical cross-sectional study with disease and control groups, who were recruited to the UK ME/ CFS Biobank [17]. Of 395 participants, 237 had been diagnosed with ME/CFS, 47 with MS, and 111 were healthy controls. This sample size is adequate to identify prevalence differences of symptoms between people with ME/CFS or MS and healthy controls, with a power of $98 \%$ and of $91 \%$ respectively at a 0.05 significance level. This is based on a conservative prevalence of $3 \%$ for cognitive symptoms, among the general population [18]. The power calculation was performed with Epi Info $7^{\mathrm{TM}}$ [19]. The UK ME/CFS Biobank participants were recruited from those with a medically confirmed diagnosis of ME/CFS from National Health Services (NHS) in London, East Anglia and other parts of the UK, covered by the Clinical Research Network and Primary Care Network (CRN/PCN), local divisions and the ME/CFS pilot Disease Register (DR). A few severely affected participants were also recruited from support groups and patient associations. Healthy controls were frequency matched to cases by gender, geographical area of residence, and age within five years of cases. Healthy controls were excluded if they had previous or current chronic fatiguing disease, such as cardiomyopathy or cancer. Healthy controls were recruited through the PCN, invited by people with ME/CFS, or responded to adverts in the same areas of recruitment. MS patients were recruited from collaborating GP practices or other secondary care health services, particularly those in the CRN/PCN located in the same region as the ME/CFS cases. Standardised procedures, including inclusion and exclusion criteria and bespoke questionnaires applied to all consenting UK ME/CFS participants, are described in detail elsewhere [20].

\section{Inclusion and exclusion criteria}

All participants, who were aged 18 to 60 years old, gave informed consent for study participation. ME/CFS cases were selected if they had a clinical diagnosis of ME/CFS according to the Centers for Disease Control and Prevention (CDC) [21] or Canadian Consensus Criteria (CCC) [8]. Disease status was confirmed by a clinical researcher, who reviewed laboratory test results to exclude alternative diagnoses. All MS cases had a diagnosis confirmed by a NHS neurologist, in compliance with the National Institute for Clinical Excellence (NICE) guidelines [22]. Information on specific type of MS was not routinely collected, but those on immunomodulatory drugs, were excluded due to the potential effects of these factors on disease processes and therefore reported symptoms. People with a diagnosis of MS were excluded if they had any other conditions apart from MS that could explain their fatigue including pregnancy or lactation.

\section{Data collection}

Data were collected from all participants using selfcompleted questionnaires including validated instruments. Others were developed specifically for the recruitment of 
participants to the UK ME/CFS Biobank, to complement the clinical assessment, which included physical examination and blood collection. Data collection ran from March 2012 to December 2015 and consisted of demographic and socio-economic data and a range of exposure variables and symptoms. Socio-demographic details collected included age, gender, ethnicity, household income, and education. Lifestyle variables included smoking and drinking habits. Symptom assessments asked about cognitive, sleep, musculoskeletal, cardiovascular, autonomic, and systemic symptoms in the week prior to participant questionnaire. Co-morbidities and family health history were also recorded. The results presented in this paper focus on cognitive and sleep symptoms.

\section{Statistical analysis}

Data were analysed using STATA 14 [23]. Cognitive symptoms analysed included problems with short-term memory, attention, and executive function; sleep disturbance was also recorded. Symptoms were analysed based on an ordinal severity scale (absent, mild, moderate, or severe) for three groups: ME/CFS, MS, and healthy controls. The prevalence of such symptoms in each group is displayed by severity. Chi-squared test was used in simple univariate analyses to compare non-continuous variables. Trends for statistical significance between symptoms across patient groups were tested with the $\chi^{2}$ test for trend and the likelihood ratio test, considering "severity" as an ordered categorical variable [24]. A cut-off of 0.05 was used for the probability of alpha-1 error to indicate significance of an association.

Various factors were investigated for potential associations with severe cognitive and sleep symptoms in ME/ CFS and MS. For the ME/CFS multivariable logistic regression model, all variables were analysed through univariate logistic regression and only included in the multivariable model if statistically significant or if considered to be clinically important. For MS, univariate logistic regression was used to investigate factors associated with severe cognitive and sleep symptoms because the small sample size precluded the use of a multivariable model.

Socio-demographic variables such as age and income were included in the final model because of a possible association between such factors and cognitive function $[25,26]$. The low numbers of ethnic minority participants led to grouping of non-white ethnicities before comparing with white participants. Alcohol consumption six months before symptoms appeared in participants with ME/CFS or MS was classified as "no drinking" or " $<14$ units/week and $\geq 14$ units/week". This cut-off of 14 units/week was chosen because it is the current guideline for maximum weekly consumption recommended by the UK Department of Health [27]. Smoking status compared non-smokers to exsmokers and current smokers. Other factors investigated in the initial univariate regression models included lifestyle factors such reported stress and activity levels as well as medical factors such as depression, anxiety, allergy, infection, asthma, and surgery in the six months before the questionnaire was completed. In the multivariable models, variables related to the time of recruitment, except for alcohol consumption and depression, which were also asked about six months previous to symptoms appearance. As duration of fatigue may have had an effect on the reported severity of symptoms, we also included this in the model, even if the difference was not statistically significant at the univariate analysis (Wilcoxon rank-sum test was used as this variable was not normally distributed). To limit the impact of missing data on the regression analysis, an assumption was made that any missing data for a specific variable corresponded to a "negative answer" or absence of the particular risk factor, providing a conservative estimate of the odds ratio. Missing data, although rare, was slightly higher in those with ME/CFS (1.42 per variable/100-persons) than those with MS (0.39 per variable/100 persons), and in healthy controls ( 0.25 per variable/100 persons).

The main dependent variable for the logistic regression analysis was a symptom severity score. This was calculated through an ordinal severity scale, with no points if the symptom was not reported, one point for a symptom reported as mild, two for moderate, and three for severe. An aggregate score (out of 27) for the severity of the nine individual cognitive and sleep symptoms was then calculated for each patient. Scores above the 75th centile for the ME/CFS group (21/27) and the MS group $(14 / 27)$ were considered to be severe for each group respectively. Logistic regression was then used to investigate factors associated with severe cognitive and sleep symptoms in each of ME/CFS and MS groups by comparing those with scores equal to or above the 75th centile with those scores below it.

\section{Results}

In the overall study population, 289 (73.2\%) were female and $106(26.8 \%)$ male. The median age was 44 years, with an interquartile range of 35 to 53 years. Three hundred and sixty-one participants (91.4\%) identified themselves as white British, and 104 (28.3\%) reported an income less than $£ 15.000$ a year at the time of data collection. Sociodemographic details of the study population are illustrated by group (Table 1 ).

Table 2 displays the prevalence of cognitive and sleep symptoms over the previous week as reported by each group, by severity. All symptoms were more prevalent in the ME/CFS group, with 'trouble concentrating' the most commonly reported symptom in this group, at $98.3 \%$. The remaining cognitive and sleep symptoms were common at over $80 \%$ for all except disorientation, which was reported by $56.5 \%$ of the ME/CFS group. Although not as frequent 
Table 1 Characteristics of study population, by group

\begin{tabular}{|c|c|c|c|c|}
\hline Variables & Categories & $\begin{array}{l}\text { ME/CFS } \\
\mathrm{n}(\%)\end{array}$ & $\begin{array}{l}\text { MS } \\
\text { n (\%) }\end{array}$ & $\begin{array}{l}\text { Healthy } \\
\text { Control n (\%) }\end{array}$ \\
\hline \multirow[t]{2}{*}{ Gender } & Male & $54(22.8)$ & $13(27.7)$ & 39 (35.1) \\
\hline & Female & $183(77.2)$ & $34(72.3)$ & $72(64.9)$ \\
\hline \multirow[t]{4}{*}{ Age group } & $<30$ & $40(16.9)$ & 0 & $18(16.2)$ \\
\hline & $30-39$ & $48(20.3)$ & $9(19.1)$ & 24 (21.6) \\
\hline & $40-49$ & $65(27.4)$ & $15(31.9)$ & $30(27.0)$ \\
\hline & $50+$ & $84(35.4)$ & $23(48.9)$ & $39(35.1)$ \\
\hline \multirow[t]{5}{*}{ Ethnicity } & White British & $220(92.3)$ & $45(95.7)$ & 96 (86.5) \\
\hline & White Other & $5(2.11)$ & $2(4.26)$ & $6(5.41)$ \\
\hline & Black African & $1(0.42)$ & 0 & $1(0.90)$ \\
\hline & Asian & $2(0.84)$ & 0 & $4(3.60)$ \\
\hline & Other & $7(2.95)$ & 0 & $4(3.60)$ \\
\hline \multirow[t]{2}{*}{ Income } & $<£ 15,000$ & $71(32.3)$ & $10(24.4)$ & $23(21.5)$ \\
\hline & $>=£ 15,000$ & $149(67.7)$ & 31 (75.6) & $84(78.5)$ \\
\hline \multirow[t]{3}{*}{ Alcohol intake ${ }^{a}$} & No Alcohol & $87(36.7)$ & $9(19.15)$ & $25(22.5)$ \\
\hline & $<14$ units/week & $118(49.8)$ & $27(57.4)$ & $69(62.1)$ \\
\hline & $\geq 14$ units/week & $32(13.5)$ & $11(23.4)$ & $17(15.3)$ \\
\hline \multirow[t]{3}{*}{ Smoking status } & Never Smoked & $150(63,2)$ & $19(40.4)$ & $78(70.2)$ \\
\hline & Ex-smoker & $62(26.1)$ & 15 (31.9) & 0 \\
\hline & Current Smoker & $25(10.5)$ & $13(27.7)$ & $33(29.8)$ \\
\hline \multirow[t]{2}{*}{ Depression $^{a}$} & No depression & $143(60.3)$ & $36(81.8)$ & $111(100)$ \\
\hline & Depression & $94(39.7)$ & $11(23.4)$ & 0 \\
\hline
\end{tabular}

${ }^{\mathrm{a} S i x}$ months before symptoms for ME/CFS or MS (or at recruitment for healthy controls)

as in ME/CFS, cognitive and sleep symptoms were widely reported in MS patients, with the most commonly reported symptoms being unrefreshing sleep (78.7\%), trouble concentrating $(74.5 \%)$, problems with sleep quality or duration $(72.3 \%)$, and short-term memory problems (72.3\%). Healthy controls reported very few symptoms.

Severe symptoms were more commonly reported in the ME/CFS group, most notably unrefreshing sleep (55.3\%) and poor quality or short duration of sleep (41.8\%). Similarly, in the MS group the most commonly reported severe symptom was unrefreshing sleep (27.7\%). The number of MS patients reporting severe non-sleep related symptoms was low ranging from 2.1 to $6.4 \%$, compared with the ME/CFS group where it ranged from 13.1 to $31.6 \%$. We have compared the duration of fatigue between people with ME/CFS (median = 11.1 years; Inter Quartile Range (IQR) 5.9 to 17.6), and people with MS (median $=10.7$ years; IQR $=6.4$ to 19.3 ), using a nonparametric test and the difference was not significant $(p=0.688)$.

\section{Logistic regression analysis}

Table 3 displays the results from the multivariable logistic regression model of the factors affecting severity of cognitive and sleep symptoms in ME/CFS patients. No clear trend could be observed between increasing age and symptom severity, although patients aged over 50 were more than three times more likely to experience severe cognitive and sleep symptoms compared with those less than 30 years old (OR 3.23, $p=0.031$ ). Current smoking increased the risk of severe cognitive and sleep symptoms by approximately three times (OR 2.93, $p=0.003$ ) and ex-smokers were more than twice as likely to experience symptoms compared with nonsmokers (OR 2.17, $p=0.034$ ). People with household incomes of $£ 15,000$ or more per year were approximately $70 \%$ less likely to report severe symptoms compared to those earning less than this (OR 0.31, $p=0.017$ ). There were no statistically significant associations between gender, ethnicity or depression and severe cognitive and sleep symptoms in ME/CFS.

Table 4 shows logistic regression analysis results for the factors affecting severity of cognitive and sleep symptoms in patients with MS. The only variable significantly associated with severe symptoms in MS was previous depression. MS patients who experienced depression prior to symptoms were over seven times more likely to report severe symptoms $(p=0.009)$. Socio-demographic variables including age, gender, ethnicity, income, smoking, and alcohol consumption were not associated with severe cognitive and sleep symptoms in MS.

\section{Discussion \\ Key findings}

Cognitive and sleep symptoms were more common in ME/CFS patients than in MS patients and healthy controls. The number of participants reporting severe symptoms was also highest in the ME/CFS group. The most commonly reported severe symptom in both groups was unrefreshing sleep: $55.3 \%$ in the ME/CFS group, compared with $27.7 \%$ in the MS group. The number of MS patients reporting severe non-sleep related symptoms was relatively low (ranging from 2.1 to $6.4 \%$ ), compared with the ME/CFS group (ranging from 13.1 to $31.6 \%$ ).

In the ME/CFS patients, multivariable logistic regression revealed that the severe cognitive and sleep symptoms were associated and increased with previous and current smoking as well as with low income $(<£ 15,000)$. Although there was no consistently significant trend with age, those over 50 years were more likely to experience severe symptoms compared with those less than 30 years old. In the MS group, univariate logistic regression demonstrated that previous depression was associated with a higher likelihood of patients reporting severe cognitive and sleep symptoms.

\section{Prevalence of severe cognitive and sleep symptoms}

The Canadian Consensus document [8] states that cognitive symptoms including confusion, slowed processing 
Table 2 Severity of cognitive and sleep symptoms over past week, by group

\begin{tabular}{|c|c|c|c|c|c|}
\hline Symptom over past week & Severity & ME/CFS n/total = $237(\%)$ & MS n/total = $47(\%)$ & Healthy control n/total = $111(\%)$ & $P$-value* \\
\hline \multirow[t]{5}{*}{ Poor sleep quality/duration } & & & & & $<0.001$ \\
\hline & Absent & $26(10.9)$ & $14(29.8)$ & $70(63.1)$ & \\
\hline & Mild & $31(13.1)$ & $15(31.9)$ & $26(23.4)$ & \\
\hline & Moderate & $81(34.2)$ & $10(21.3)$ & $13(11.7)$ & \\
\hline & Severe & $99(41.8)$ & $8(17.0)$ & $2(1.8)$ & \\
\hline \multirow[t]{5}{*}{ Unrefreshing sleep } & & & & & $<0.001$ \\
\hline & Absent & $11(4.6)$ & $10(21.3)$ & $64(57.7)$ & \\
\hline & Mild & $22(9.3)$ & $12(25.5)$ & $34(30.6)$ & \\
\hline & Moderate & $73(30.8)$ & $12(25.5)$ & $11(9.9)$ & \\
\hline & Severe & $131(55.3)$ & $13(27.7)$ & $2(1.8)$ & \\
\hline \multirow[t]{5}{*}{ Difficulty retaining information } & & & & & $<0.001$ \\
\hline & Absent & $21(8.9)$ & $16(34.0)$ & $93(83.8)$ & \\
\hline & Mild & $70(29.5)$ & $17(36.2)$ & $18(16.2)$ & \\
\hline & Moderate & $83(35.0)$ & $11(23.4)$ & 0 & \\
\hline & Severe & $63(26.6)$ & $3(6.4)$ & 0 & \\
\hline \multirow[t]{5}{*}{ Difficulty understanding/thinking clearly } & & & & & $<0.001$ \\
\hline & Absent & $27(11.4)$ & $23(48.9)$ & $97(87.4)$ & \\
\hline & Mild & $77(32.5)$ & $12(25.5)$ & $14(12.6)$ & \\
\hline & Moderate & $76(32.1)$ & $9(19.1)$ & 0 & \\
\hline & Severe & $57(24.1)$ & $3(6.4)$ & 0 & \\
\hline \multirow[t]{5}{*}{ Slow thinking } & & & & & $<0.001$ \\
\hline & Absent & $34(14.3)$ & $19(40.4)$ & $97(87.4)$ & \\
\hline & Mild & $77(32.5)$ & $14(29.8)$ & $14(12.6)$ & \\
\hline & Moderate & $80(33.8)$ & $11(23.4)$ & 0 & \\
\hline & Severe & $46(19.4)$ & $3(6.4)$ & 0 & \\
\hline \multirow[t]{5}{*}{ Disorientation } & & & & & $<0.001$ \\
\hline & Absent & $107(45.1)$ & $31(66.0)$ & $107(96.4)$ & \\
\hline & Mild & $51(21.5)$ & $9(19.1)$ & $3(2.7)$ & \\
\hline & Moderate & $48(20.3)$ & $6(12.8)$ & $1(0.9)$ & \\
\hline & Severe & $31(13.1)$ & $1(2.1)$ & 0 & \\
\hline \multirow[t]{5}{*}{ Trouble concentrating } & & & & & $<0.001$ \\
\hline & Absent & $6(2.5)$ & $12(25.5)$ & $84(75.7)$ & \\
\hline & Mild & $54(22.8)$ & $15(31.9)$ & $23(20.7)$ & \\
\hline & Moderate & $102(43.0)$ & $18(38.3)$ & $3(2.7)$ & \\
\hline & Severe & $75(31.6)$ & $2(4.3)$ & $1(0.9)$ & \\
\hline \multirow[t]{5}{*}{ Short term memory problems } & & & & & $<0.001$ \\
\hline & Absent & $22(9.3)$ & $13(27.7)$ & $90(81.1)$ & \\
\hline & Mild & $65(27.4)$ & $17(36.2)$ & $19(17.1)$ & \\
\hline & Moderate & $91(38.4)$ & $15(31.9)$ & $2(1.8)$ & \\
\hline & Severe & $59(24.9)$ & $2(4.3)$ & 0 & \\
\hline \multirow[t]{5}{*}{ Brain fog/confusion } & & & & & $<0.001$ \\
\hline & Absent & $38(16.0)$ & $21(44.7)$ & $99(89.2)$ & \\
\hline & Mild & $62(26.2)$ & $9(19.1)$ & $11(9.9)$ & \\
\hline & Moderate & $77(32.5)$ & $15(31.9)$ & $1(0.9)$ & \\
\hline & Severe & $60(25.3)$ & $2(4.3)$ & 0 & \\
\hline
\end{tabular}


Table 3 Multivariable Analysis: Factors related to prevalence of severe cognitive and sleep symptoms in ME/CFS

\begin{tabular}{llll}
\hline Variable & Odds ratio & $95 \%$ Confidence interval \\
\hline Age $<30$ & 1.00 & - \\
Age $30-39$ & 2.30 & $0.69-7.66$ \\
Age $40-49$ & 2.34 & $0.73-7.56$ \\
Age $50+$ & 3.23 & $1.04-10.04$ \\
Male & 1.00 & - \\
Female & 1.88 & $0.81-4.30$ \\
Non-white ethnicity & 1.00 & - \\
White ethnicity & 0.65 & $0.18-2.39$ \\
Income $<£ 15,000$ & 1.00 & - \\
Income $>$ £15,000 & 0.31 & $0.12-0.81$ \\
No alcohol (six months before) & 1.00 & - \\
Alcohol $<14$ units/week (six months before disease onset) & 0.96 & $0.48-1.94$ \\
Alcohol $\geq 14$ units/week (six months before disease onset) & 1.15 & $0.40-3.30$ \\
No smoking & 1.00 & - \\
Ex-smoker & 2.17 & $1.06-4.42$ \\
Current smoker & 2.93 & $1.11-7.70$ \\
No depression before symptoms & 1.00 & - \\
Depression before symptoms & 1.58 & 0.017 \\
Duration of fatigue (years) & 0.99 & $0.82-3.03$ \\
\hline
\end{tabular}

of information, difficulty concentrating, poor short-term memory, and sleep problems are common in ME/CFS. Fukuda et al. [28] propose that apart from fatigue and musculoskeletal pain, the most prominent features of $\mathrm{ME} / \mathrm{CFS}$ are impairments in concentration, short-term memory, and sleep disturbances. Recently, the Institute of Medicine reviewed the evidence on the major symptoms of ME/CFS and reiterated that cognitive symptoms, sleep related symptoms and primary sleep disorders are common features [1]. Our study provides an empirical estimation of the proportion of ME/CFS patients affected by cognitive symptoms, with all symptoms except disorientation being reported in more than $80 \%$ of ME/CFS patients. We also found that the classic

Table 4 Univariate analysis: factors related to the prevalence of severe cognitive and sleep symptoms in MS

\begin{tabular}{lll}
\hline Variable & Odds Ratio & $95 \%$ confidence interval \\
\hline Age $<30$ & 1.00 & - \\
Age $30-39$ & 0.94 & $0.18-4.79$ \\
Age $40+$ & 0.47 & $0.10-2.16$ \\
Male & 1.00 & - \\
Female & 0.58 & $0.15-2.23$ \\
Income $<£ 15,000$ & 1.00 & - \\
Income $>=£ 15,000$ & 0.67 & $0.05-8.64$ \\
No alcohol (six months before) & 1.00 & - \\
Alcohol $<14$ units/week (six months before disease onset) & 0.53 & $0.11-2.49$ \\
Alcohol $\geq 14$ units/week (six months before disease onset) & 0.28 & $0.04-2.09$ \\
No smoking & 1.00 & - \\
Ex-smoker & 0.79 & $0.18-3.53$ \\
Current smoker & 0.96 & $0.21-4.42$ \\
No depression before symptoms & 1.00 & - \\
Depression before symptoms & 7.25 & $1.65-31.8$ \\
\hline
\end{tabular}


ME/CFS symptoms of sleep disturbance and trouble concentrating were most commonly reported as severe compared to other symptoms.

Unrefreshing sleep, or sleep of poor quality or short duration were the most commonly reported severe symptoms in both the ME/CFS and MS groups. Krupp et al. evaluated the sleep characteristics in 72 patients with ME/CFS compared with 57 MS patients [11] and found that the ME/CFS patients experienced significantly more sleep disturbance compared with MS patients. Among the ME/ CFS patients, 37\% reported light sleeping, 15\% reported inadequate sleep, and $27 \%$ reported early waking, compared with 10,5 , and $0 \%$ respectively for the MS group. The findings of our study are consistent with rates were higher for ME/CFS participants, although sleep disturbances were common in both groups.

Guidelines for ME/CFS participant (Fukuda criteria) study inclusion advise excluding those with primary sleep disorders. Unrefreshing sleep is the only ME/ CFS criteria relating to sleep in the Fukuda guidelines [28]. However, other authors argue that primary sleep disorders should be treated as co-morbidities, and their presence should not preclude a diagnosis of ME/CFS [1]. In people with ME/CFS, no consistent abnormal sleep pattern has been found on polysomnography [29] adding to the difficulty in treating sleep disturbances. Nevertheless, the emergence of new, more sensitive techniques suggest that alterations in sleep stage transitions and other physiological mechanisms, such as heart rate variability and cortisol profiles, may be evident in ME/CFS. A 2013 crosssectional study of $343 \mathrm{ME} / \mathrm{CFS}$ patients [29] described four sleep-specific phenotypes, with objective differences among them in sleep onset latencies as well as rapid eye movement (REM) stages and latencies. The high prevalence of severe sleep symptoms reported in our study indicates that data characterising distinct sleep patterns could prove vital in identifying targeted methods for treatment in ME/CFS patients.

\section{Risk factors for severe cognitive and sleep symptoms}

Our study is unique in exploring risk factors for cognitive and sleep symptoms and their severity in ME/CFS. A 2008 systematic review of eleven case-control, cohort, and cross-sectional studies assessed various potential factors correlated with the development of ME/CFS, but causal evidence is lacking [30]. Factors found to be significantly associated with the development of ME/CFS, which our study also investigated, were older age [31, 32], being female [32, 33], the presence of an anxiety disorder [31], mood disorder [34] or stress [35], and a history of allergies or asthma [36]. Based on our findings, the majority of these factors do not appear to translate from risk of development of ME/CFS to risk of severe cognitive and sleep symptoms in ME/CFS. That said, ME/CFS patients over the age of 50 were more than three times more likely to experience severe cognitive and sleep symptoms, compared to those under age 30. The reason for older age causing more severe symptoms are complex, but may be at least partially due to age-related decreases in brain dopamine activity contributing to impaired performance on tasks that involve frontal brain regions [37]. The pathophysiological processes occurring in ME/CFS may exaggerate normal age-related processes. For instance, Xenon-computed tomography blood flow studies show significantly lower cortical/cerebellar regional cerebral blood flow (rCBF) in ME/CFS [38].

A cross-sectional population-based US study $(n=5623)$ found that income was not related to the prevalence of $\mathrm{ME} / \mathrm{CFS}$ in the general population [39]. However, our analysis found that ME/CFS participants with household incomes of under $£ 15,000$ per year were at an increased risk of severe cognitive and sleep symptoms. Although there scant literature exploring the effect of income on severity of symptoms in ME/CFS, a 1995 cohort study [40] of 49 patients found that environmental and psychosocial stressors exacerbated self-reported ME/CFS symptoms following a natural disaster. It is plausible that such stressors may play a similar role in the daily lives of individuals with lower incomes. In addition, existing literature describes an association between relative poverty and sleep symptoms in healthy individuals [41]. However, more evidence is required to explore the possible association between low income and severity of cognitive symptoms in ME/CFS, and the precise mechanism of this relationship.

Smoking was also found to be associated with severe cognitive and sleep symptoms. Previous evidence from a large multicentre cohort study (EURODEM), with 9209 non-demented patients selected for follow-up, found that smoking leads to cognitive decline [42]. The minimental state exam (MMSE) of those who never smoked declined by 0.03 points/year. The decline in score for previous smokers was 0.03 points greater than this, and for current smokers was 0.13 points greater than never smokers $(p<0.001)$. The results of our study concur with such evidence, finding a relationship between smoking habits and severe cognitive and sleep symptoms in ME/ CFS. This is of clinical importance as it suggests that stopping or preventing smoking may help reduce the risk of developing more severe symptoms in such patients.

\section{Strengths and limitations}

This study included a relatively large sample size of ME/ CFS participants $(n=237)$, a healthy control group, and participants with another neurological disease for comparison. This enabled a reliable statistical analysis of predictive factors for people with severe symptoms of 
ME/CFS. The study population included participants from multiple sites across the UK and with varying degrees of severity, so although our results may not be generalizable to the entire UK population, they are reflective of a wide range of participants rather than a particular subset. All data were collected in a standardized form and all participants had contact with the clinical team for assessment, optimizing data reliability. Finally, data were gathered on a broad range of sociodemographic variables, exposures, and symptoms. For this reason, we have been able to explore the prevalence of cognitive and sleep symptoms between different disease groups and investigate numerous predictive factors.

The main limitation of the study lies in the relatively low number of participants with MS $(n=47)$, restricting our ability to conduct multivariable analysis in this group. Nonetheless, univariate regression analysis was used to explore potentially associated factors of clinical importance, but these will require validation in studies with larger sample sizes. In addition, information on specific type of MS was not routinely collected, and therefore we were not able to ascertain differences between distinct MS subtypes. In the overall study population, most of the participants recruited identified themselves as being of white British ethnicity $(n=391)$, restricting the generalizability of results to the wider multi-ethnic population. Although our analysis included severely-affected participants there may have been some people with ME/CFS who were so ill, for example on nasogastric feeding, that they could not participate in the study. Additional limitations inherent to the study design and data collection methods are recognized and have to be taken into account when considering our findings, such as the possibility of residual confounding. The cross-sectional nature of the data, even with a control group, can be indicative of associations, but not causality. Additionally, recall bias is also an issue to be considered in self-reported data collection. However, the consistency of responses across the three groups indicates that this bias is unlikely to have been significant.

\section{Implications for clinical practice and future research}

In MS patients, a history of depression was found to be associated with severe cognitive and sleep symptoms. Depression is common in MS [43] and is thought to interfere with cognitive function. Arnett et al. [44] conducted a critical review of the existing literature on depression in MS, to find that a positive link has been consistently noted between depression and cognitive functioning. Depression affects many aspects of cognitive functioning in MS, including working memory, processing speed, and executive functioning [45-47]. Our findings therefore support existing evidence, and suggest that efforts aiming to treat or prevent depression could lead to improvements in the reported severity of cognitive symptoms in patients with MS.

The high prevalence of cognitive and sleep symptoms in ME/CFS highlights the need for improved strategies to combat their effects. Unlike MS, the severity of these symptoms seems to be unrelated to depression, and might reflect more generalised and subtle abnormalities in the CNS. Unrefreshing sleep was the most commonly reported severe symptom. Research has demonstrated that sleep physiology is altered in ME/CFS patients [48], but management of the condition, and available pharmacological therapy, still fails to address this critical issue. Future research must explore both pharmacological and non-pharmacological methods aimed at improving quality and duration of sleep in ME/CFS.

In clinical practice, patients may be advised of the various factors that can lead to severe cognitive and sleep symptoms. To recommend smoking cessation is a general public health measure that can be reinforced, and prevention of smoking in those at potential higher risk of $\mathrm{ME} / \mathrm{CFS}$, such as those with prolonged fatigue following an infectious disease, is important to reduce risk of severe cognitive and sleep symptoms. Public health and medical interventions to improve symptoms must recognise that those with a low-income $(\leq £ 15,000)$ seem to be at a higher risk of developing severe symptoms.

Despite the relatively high prevalence of cognitive impairment in MS, cognitive function is not assessed routinely in clinical practice or in clinical trials [49]. Our findings suggest that sleep problems, rather than other cognitive problems, are severe in a large minority of MS patients. The perception that cognitive assessments are costly, time-consuming and difficult to interpret has contributed to the failure to incorporate cognitive testing into standard clinical evaluation of patients with MS. Detailed studies of cognitive impairment in MS are rare, and guidelines for the assessment of cognitive or sleep function in MS are lacking. Future research must address these gaps in the current knowledge base. Researchers may find it beneficial to incorporate cognitive and sleep function measures into clinical assessment of MS and ME/CFS, and encourage the development of novel therapies targeting such symptoms.

\section{Conclusions}

Cognitive and sleep symptoms were more common in people with ME/CFS in this study than in both healthy controls and people with MS. This provides further support for existing evidence of central nervous system abnormalities in ME/CFS and highlights the need for improved strategies to address the effects of such symptoms. Unrefreshing sleep was the most commonly reported severe symptom in both people with ME/CFS and people with MS, although it was more prevalent in 
those with ME/CFS. Our findings suggest that people with $\mathrm{ME} / \mathrm{CFS}$ who are smokers, or have a low income, are more likely to report severe cognitive and sleep symptoms. Future research should aim to develop strategies to prevent the progression of severe cognitive and sleep symptoms through early interventions that prioritise patients identified as being at highest risk.

\begin{abstract}
Abbreviations
CCC: Canadian Consensus Criteria; CDC: Centers for Disease Control and Prevention; CRN/PCN: Clinical Research Network and Primary Care Network; DR: Disease register; IQR: Interquartile range; LSHTM: London School of Hygiene \& Tropical Medicine; ME/CFS: Myalgic encephalomyelitis/chronic fatigue syndrome; MMSE: Mini-mental state exam; MS: Multiple sclerosis; NHS: National Health Service; NICE: National Institute for Clinical Excellence; NRES: National Research Ethics Service; OR: Odds ratio; rCBF: Regional cerebral blood flow; REM: Rapid eye movement
\end{abstract}

\section{Acknowledgements}

We would like to express our sincere appreciation to the many people both those with and without ME/CFS - who have so generously contributed to the UK ME/CFS Biobank by donating their time, resources, and precious energy to participate in the project. We would also like to thank CURE-ME team member Erinna Bowman for her valuable contribution to this work.

\section{Funding}

The UK ME/CFS Biobank was established with a joint grant from the UK charities Action for M.E., the ME Association, ME Research UK, and a private donor. Research reported in this publication was supported by the National Institute Of Allergy And Infectious Diseases of the National Institutes of Health under Award Number R01Al103629. The content is solely the responsibility of the authors and does not necessarily represent the official views of the National Institutes of Health.

\section{Availability of data and materials}

The datasets generated during and/or analysed during the current study are part of the UK ME/CFS Biobank, and will be available for researchers upon a signed Data Transfer Agreement.

\section{Authors' contributions}

$J V, L N$ and EL were involved in the design, analysis and interpretation, and reporting of this project. AA helped in the design, data collection, and data analysis. CK contributed to the design, data collection and revision of the manuscript. All authors have read and approved the final manuscript for publication.

\section{Competing interests}

The authors declare that they have no competing interests.

\section{Consent for publication}

Consent for publication has been obtained from all participants and consent forms can be provided if required.

\section{Ethics approval and consent to participate}

This study is a partial analysis of the clinical dataset from the UK ME/CFS Biobank, included in the broader proposal, which received ethical approval from the London School of Hygiene \& Tropical Medicine (LSHTM) Ethics Committee (Ref. 6123) and National Research Ethics Service (NRES) LondonBloomsbury Research Ethics Committee (REC ref. 11/10/1760, IRAS ID: 77,765). All participants provided written informed consent.

\section{Publisher's Note}

Springer Nature remains neutral with regard to jurisdictional claims in published maps and institutional affiliations.

\section{Author details}

'Guy's Campus, King's College London School of Medicine, London SE1 1UL, UK. ${ }^{2}$ University of California, San Francisco, School of Medicine, San Francisco, CA 94143, USA. ${ }^{3}$ Faculty of Infectious and Tropical Diseases, CURE-ME Team,
London School of Hygiene \& Tropical Medicine Disability and Eye Health Group, International Centre for Evidence in Disability (ICED, K/490, Keppel St, London WC1E 7HT, UK.

Received: 11 September 2016 Accepted: 12 June 2017

Published online: 20 June 2017

\section{References}

1. Clayton EW. Beyond myalgic encephalomyelitis/chronic fatigue syndrome: an IOM report on redefining an illness. JAMA. 2015;313(11):1101-2.

2. McDonald WI, Compston A, Edan G, Goodkin D, Hartung H, Lublin FD, et al. Recommended diagnostic criteria for multiple sclerosis: guidelines from the international panel on the diagnosis of multiple sclerosis. Ann Neurol. 2001;50(1):121-7.

3. Ablashi D, Eastman H, Owen C, Roman M, Friedman J, Zabriskie J, et al. Frequent HHV-6 reactivation in multiple sclerosis (MS) and chronic fatigue syndrome (CFS) patients. J Clin Virol. 2000;16(3):179-91.

4. Chaudhuri A, Behan PO. Neurological dysfunction in chronic fatigue syndrome. J Chronic Fatigue Syndrome. 2000;6(3-4):51-68.

5. White AT, Light AR, Hughen RW, Vanhaitsma TA, Light KC. Differences in metabolite-detecting, adrenergic, and immune gene expression after moderate exercise in patients with chronic fatigue syndrome, patients with multiple sclerosis, and healthy controls. Psychosom Med. 2012;74(1):46-54.

6. Robertson MJ, Schacterle R, Mackin G, Wilson S, Bloomingdale K, Ritz J, et al. Lymphocyte subset differences in patients with chronic fatigue syndrome, multiple sclerosis and major depression. Clinical \& Experimental Immunology. 2005;141(2):326-32.

7. Frykholm B. On the question of infectious aetiologies for multiple sclerosis, schizophrenia and the chronic fatigue syndrome and their treatment with antibiotics. Med Hypotheses. 2009;72(6):736-9.

8. Carruthers BM, Jain AK, De Meirleir KL, Peterson DL, Klimas NG, Lerner AM, et al. Myalgic encephalomyelitis/chronic fatigue syndrome: clinical working case definition, diagnostic and treatment protocols. J Chronic Fatigue Syndrome. 2003;11(1):7-115.

9. Jongen PJ, Ter Horst AT, Brands AM. Cognitive impairment in multiple sclerosis. Minerva Med. 2012;103(2):73-96.

10. Logsdon RG, Gibbons LE, McCurry SM, Teri L. Assessing quality of life in older adults with cognitive impairment. Psychosom Med. 2002;64(3):510-9.

11. Krupp LB, Jandorf L, Coyle P, Mendelson W. Sleep disturbance in chronic fatigue syndrome. J Psychosom Res. 1993;37(4):325-31.

12. Kaynak H, Altintaş A, Kaynak D, Uyanik Ö, Saip S, Ağaoğlu J, et al. Fatigue and sleep disturbance in multiple sclerosis. Eur J Neurol. 2006;13(12):1333-9.

13. Morris G, Maes M. Myalgic encephalomyelitis/chronic fatigue syndrome and encephalomyelitis disseminata/multiple sclerosis show remarkable levels of similarity in phenomenology and neuroimmune characteristics. BMC Med. 2013;11:205-7015-11-205

14. Maes M. Inflammatory and oxidative and nitrosative stress pathways underpinning chronic fatigue, somatization and psychosomatic symptoms. Current opinion in psychiatry. 2009;22(1):75-83.

15. Uveges JM, Parker JC, Smarr KL, McGowan JF, Lyon MG, Irvin WS, et al. Psychological symptoms in primary fibromyalgia syndrome: relationship to pain, life stress, and sleep disturbance. Arthritis \& Rheumatism. 1990; 33(8):1279-83.

16. McClintock SM, Husain MM, Greer TL, Cullum CM. Association between depression severity and neurocognitive function in major depressive disorder: a review and synthesis. Neuropsychology. 2010;24(1):9.

17. London School of Hygiene \& Tropical Medicine. The UK ME-CFS Biobank. 2015; Available at: http://cureme.Ishtm.ac.uk/. Accessed 22 Apr 2016.

18. DeCarli C. Mild cognitive impairment: prevalence, prognosis, aetiology, and treatment. The Lancet Neurology. 2003;2(1):15-21.

19. Centers for Disease Control and Prevention. Epi Info User Guide. 2016; Available at: https://wwwn.cdc.gov/epiinfo/user-guide/StatCalc/How-toOpening-StatCalc.html. Accessed 22 Jul 2016.

20. Lacerda EM, Bowman EW, Cliff JM, Kingdon CC, King EC, Lee JS, et al. The UK ME/CFS Biobank for biomedical research on Myalgic Encephalomyelitis/ Chronic Fatigue Syndrome (ME/CFS) and Multiple Sclerosis. Open Journal of Bioresources 2017 In Press.

21. Centres for Disease Control and Prevention. Chronic fatigue syndrome. 1994; Available at: http://www.cdc.gov/cfs/case-definition/1994.html. Accessed 29 Mar 2016 
22. Baker R, Shaw E. Guidelines: diagnosis and management of chronic fatigue syndrome or myalgic encephalomyelitis (or encephalopathy): summary of NICE guidance. BMJ: British Medical Journal. 2007;335(7617):446-8.

23. StataCorp. Stata statistical software: release 14. 2015.

24. Kirkwood BR, Sterne JA. Essential medical statistics. Wiley; 2010.

25. Boyede GO, Lesi FE, Ezeaka VC, Umeh CS. Impact of sociodemographic factors on cognitive function in school-aged HIV-infected Nigerian children. HIV AIDS (Auckl). 2013;5:145-52.

26. Einarsson U, Gottberg K, von Koch L, Fredrikson S, Ytterberg C, Jin YP, et al. Cognitive and motor function in people with multiple sclerosis in Stockholm County. Mult Scler. 2006;12(3):340-53.

27. Department of Health. UK chief medical Officers' alcohol guidelines review: summary of the proposed new guidelines. 2016; Available at: https://www. gov.uk/government/uploads/system/uploads/attachment_data/file/489795/ summary.pdf. Accessed 20 May 2016.

28. Fukuda K, Straus SE, Hickie I, Sharpe MC, Dobbins JG, Komaroff A. The chronic fatigue syndrome: a comprehensive approach to its definition and study. Ann Intern Med. 1994;121(12):953-9.

29. Gotts ZM, Deary V, Newton J, Van der Dussen D, De Roy P, Ellis JG. Are there sleep-specific phenotypes in patients with chronic fatigue syndrome? A cross-sectional polysomnography analysis. BMJ Open. 2013;3(6):e002999. doi:10.1136/bmjopen-2013-002999.

30. Hempel S, Chambers D, Bagnall A, Forbes C. Risk factors for chronic fatique syndrome/myalgic encephalomyelitis: a systematic scoping review of multiple predictor studies. Psychol Med. 2008;38(7):915-26.

31. Chalder T, Goodman R, Wessely S, Hotopf M, Meltzer H. Epidemiology of chronic fatigue syndrome and self reported myalgic encephalomyelitis in 515 year olds: cross sectional study. BMJ. 2003;327(7416):654-5.

32. Huibers MJ, Bleijenberg G, van Amelsvoort LG, Beurskens AJ, van Schayck $C P$, Bazelmans $E$, et al. Predictors of outcome in fatigued employees on sick leave: results from a randomised trial. J Psychosom Res. 2004;57(5):443-9.

33. Viner R, Hotopf M. Childhood predictors of self reported chronic fatigue syndrome/myalgic encephalomyelitis in adults: national birth cohort study. BMJ. 2004;329(7472):941.

34. White PD, Thomas JM, Kangro HO, Bruce-Jones WD, Amess J, Crawford DH, et al. Predictions and associations of fatigue syndromes and mood disorders that occur after infectious mononucleosis. Lancet. 2001;358(9297):1946-54.

35. Kato K, Sullivan PF, Evengård B, Pedersen NL. Premorbid predictors of chronic fatigue. Arch Gen Psychiatry. 2006;63(11):1267-72.

36. Bell KM, Cookfair D, Bell DS, Reese P, Cooper L. Risk factors associated with chronic fatigue syndrome in a cluster of pediatric cases. Review of Infectious Diseases. 1991;13(Supplement 1):S32-8.

37. Volkow ND, Gur RC, Wang G, Fowler JS, Moberg PJ, Ding Y, et al. Association between decline in brain dopamine activity with age and cognitive and motor impairment in healthy individuals. Am J Psychiatry. 1998;155(3):344-9.

38. Yoshiuchi K, Farkas J, Natelson BH. Patients with chronic fatigue syndrome have reduced absolute cortical blood flow. Clin Physiol Funct Imaging. 2006;26(2):83-6.

39. Reeves WC, Jones JF, Maloney E, Heim C, Hoaglin DC, Boneva RS, et al. Prevalence of chronic fatigue syndrome in metropolitan, urban, and rural Georgia. Popul Health Metrics. 2007;5(1):1.

40. Lutgendorf SK, Antoni MH, Ironson G, Fletcher MA, Penedo F, Baum A, et al. Physical symptoms of chronic fatigue syndrome are exacerbated by the stress of hurricane Andrew. Psychosom Med. 1995;57(4):310-23.

41. Grandner MA, Petrov M, Rattanaumpawan P, Jackson N, Platt A, Patel NP. Sleep symptoms, race/ethnicity, and socioeconomic position. J Clin Sleep Med. 2013;9(9):897-905.

42. Ott A, Andersen K, Dewey ME, Letenneur L, Brayne C, Copeland JR, et al. Effect of smoking on global cognitive function in nondemented elderly. Neurology. 2004;62(6):920-4.

43. Minden SL, Schiffer RB. Depression and mood disorders in multiple sclerosis. Cogn Behav Neurol. 1991;4(1):62-77.

44. Arnett PA, Barwick FH, Beeney JE. Depression in multiple sclerosis: review and theoretical proposal. J Int Neuropsychol Soc. 2008;14(05):691-724.

45. Arnett PA, Higginson Cl, Voss WD, Bender WI, Wurst JM, Tippin JM. Depression in multiple sclerosis: relationship to working memory capacity. Neuropsychology. 1999;13(4):546.

46. Arnett PA, Higginson Cl, Voss WD, Wright B, Bender WI, Wurst JM, et al. Depressed mood in multiple sclerosis: relationship to capacity-demanding memory and attentional functioning. Neuropsychology. 1999;13(3):434.
47. Denney DR, Lynch SG, Parmenter BA, Horne N. Cognitive impairment in relapsing and primary progressive multiple sclerosis: mostly a matter of speed. J Int Neuropsychol Soc. 2004;10(07):948-56.

48. Whelton CL, Salit I, Moldofsky H. Sleep, Epstein-Barr virus infection, musculoskeletal pain, and depressive symptoms in chronic fatigue syndrome. J Rheumatol. 1992;19(6):939-43.

49. Patti F. Cognitive impairment in multiple sclerosis. Mult Scler. 2009;15(1):2-8.

\section{Submit your next manuscript to BioMed Central and we will help you at every step:}

- We accept pre-submission inquiries

- Our selector tool helps you to find the most relevant journal

- We provide round the clock customer support

- Convenient online submission

- Thorough peer review

- Inclusion in PubMed and all major indexing services

- Maximum visibility for your research

Submit your manuscript at www.biomedcentral.com/submit
) Biomed Central 